\title{
Redes sociales: nuevos espacio de disputa y confrontación. Un análisis del 30-S en Ecuador
}

\section{Social networks: new spaces for dispute and confrontation. An analysis of the 30-S at Ecuador}

DOI: https://doi.org/10.29166/tyc.v1i20.1896

\begin{abstract}
Valeria Yarad Jeada
Es Ph.D. en Sociología y Antropología, máster en Metodología de la Investigación en CCSS por la Universidad Complutense de Madrid, y licenciada en Comunicación Social por la Universidad Central del Ecuador. Tiene más de nueve años de experiencia en el ámbito de la investigación y la comunicación, así como la docencia universitaria. Actualmente, desarolla consultorías como investigadora independiente.
\end{abstract}

Correo: vale.yarad@gmail.com

\section{Resumen}

Este trabajo muestra una etnografía virtual en Facebook y Twitter durante la revuelta policial, conocida como el 30-S de 2010 en Ecuador. Sobre esos acontecimientos se ha planteado la hipótesis de un intento de golpe de Estado contra el entonces presidente, Rafael Correa. Los usuarios de las redes sociales fueron protagonistas frente a los medios tradicionales. Para este trabajo, se seleccionaron datos abiertos ${ }^{1}$ y se analizaron de manera cualitativa los discursos de los internautas.

Palabras clave: 30S, Facebook, Twitter, etnografía virtual.

\section{Abstract}

This work shows a virtual ethnography on Facebook and Twitter throughout the police revolt in Ecuador, known as 30-S 2010. On these events, the hypothesis of an attempted coup against the President, Rafael Correa, has been raised. Users of social networks were protagonists compared to traditional media. For this work, open data was selected and the speeches of Internet users were analyzed qualitatively.

Keywords: 30S, Facebook, Twitter, virtual ethnography.

1 Todos los perfiles de Twitter y Facebook que se exponen en el artículo son abiertos, es decir no tienen restricción alguna, por ende son contenido público que se puede citar y referenciar. 


\section{Introducción}

Como contexto, según el boletín oficial de la Agencia de Regulación y Control de las Telecomunicaciones (Arcotel) ${ }^{2}$, el acceso a internet móvil en Ecuador alcanzó cuotas de alrededor del 53,41\% hasta el año 2018. A medida que el acceso a internet crece, se nota un cambio en el consumo de la información, así como en el uso de estos medios como espacios de protesta y organización social. Ecuador se caracteriza por la debilidad de sus instituciones, lo que se refleja en tres derrocamientos presidenciales en menos de 10 años (entre 1996 y 2005), y las protestas siempre han tenido lugar en las calles con el seguimiento directo de los medios de comunicación.

A medida que pasa el tiempo y gracias al acceso a internet, fijo y móvil, así como a la penetración los denominados teléfonos inteligentes, como uno de los dispositivos más usados, se desarrolla una suerte de activismo en estos espacios. Hill y Hughes (1998) se preguntan si internet va a remplazar a la televisión como el principal medio de comunicación política. Estos autores ya vaticinaron al "nuevo medio" como una vía para generar conciencia política

Es así que, casi 20 años después de los postulados de Hill y Hughes, se observan en la red revueltas de grupos opositores a regímenes de corte opresor. El ejemplo más representativo fue la "Primavera Árabe", que se originó en Túnez en 2010 (Tavarés y Pineda, 2018, p. 41) y que fue el inicio de otros sucesos similares países del Medio Oriente, como Egipto, Irán, Siria, entre otros.

Actualmente se habla del concepto democracia 2.0 (Caldevilla, 2009) que representa aquellos procesos en los que la red ha configurado esa nueva forma de la opinión pública (De la Cueva, 2018) mediante la libertad de expresión que ejercen los individuos que antes no tenían posibilidades de expresar su voz. Se genera así una ruptura de los paradigmas en lo que se refiere a los medios de comunicación y a la comunicación de masas (Eco, 1999; Mattelart y Mattelart, 1997).

Tavarés y Pineda (2018, p.42) argumentan que el uso de estos espacios busca la emancipación y la participación política como extensión de los espacios reales. Internet, creado por los servicios de inteligencia militar estadounidenses (Castells, 1999) y que en la actualidad forma parte del mundo laboral y de gestión de las rutinas personales, se ha convertido en un espacio de reivindicación política, excepto en algunos países donde predominan las dictaduras y en los que las redes sociales y el acceso a internet está restringido por el propio sistema político.

La tecnología y las redes sociales ya no son elementos aislados, ni accesorios de la vida social, aunque representen un estilo de vida vinculado al consumo (computador, teléfono inteligente, tableta, etc.). Se trata de procesos de interacción entre la máquina y los sujetos, así como de coproducción (Aibar, 1996), lo que representa una relación simbiótica, entre la tecnología y la sociedad. Siguiendo a Latour (2005) los seres humanos desarro-

2 Estudio disponible en: http://www.arcotel.gob.ec/estadisticas-2/. Último acceso: 29 de enero de 2019 
llan una apropiación tecnológica en la que los usuarios adaptan a sus necesidades (familiares, sociales, culturales, educativos, laborales, etc.) las formas en que utilizan la red.

Uno de los postulados de una de las escuelas más destacadas en los estudios sobre tecnología, el Actor Network Theory, con Bruno Latour como uno de sus principales exponentes, señala que los procesos de desarrollo tecnológico tienen un fuerte componente social y cultural y, por tanto, son esencialmente conflictivos y cargados de situaciones impredecibles. Además, dice, los sujetos imprimen en estos usos una dirección y una intención.

La comunicación humana es discursiva en sí misma, la palabra representa y reproduce esas ideas sociales o individuales que persiguen los sujetos. Desde la masificación de la internet, las dinámicas comunicacionales han cambiado. Internet se transforma de un sistema de comunicación militar a una herramienta empresarial, para luego insertarse en los espacios de ocio-entretenimiento y, paulatinamente, se convierte en uno de los espacios destacados de las relaciones sociales mediante la interacción entre las personas. Estas interacciones que, en los inicios de internet, se enfocaban más en lo casual, pasan a formar parte de los entornos políticos (Lozada, 2004), de reivindicación y defensa.

Manuel Castells (2000) dice que el sentimiento de indignación es el principal factor que ayuda a generar un empoderamiento de los actores individuales que se convierten en actores sociales. El proceso que menciona Castells (2000) sirve para que estos sujetos, muchos de ellos alejados del quehacer político, usen los medios digitales como forma de expresión a favor o en contra de un determinado accionar político, social, ideológico, cultural, etc.

La palabra "indignación" ha sido la protagonista en distintos momentos políticos, como el 15-M, en España, y Ocupy Wall Street, en Estados Unidos, en cuyos escenarios los jóvenes y adultos empezaron a protestar en contra del sistema político y económico, al que calificaban de desigual, injusto y opresor.

Estas protestas, a diferencia de años anteriores, tienen su punto de partida en las redes sociales. Gil y Guilleumas (2017) analizaron más de 17.000 tweets de usuarios que se conectaron por Twitter para organizar y convocar la protesta del 15-M en las distintas plazas públicas de España. Es así que la red empieza a ser usada además como una plataforma que favorece el activismo político.

Internet se convierte así en ese mundo no censurable, donde nada (o casi nada) se puede controlar, pese a que la vigilancia de estos entornos es algo común, sea por razones de seguridad, comerciales, empresariales, etc. (Bauman y Lyon, 2013).

En palabras de Campos Freire (2008) las redes sociales han trastocado los medios tradicionales. Los usuarios dejan de ser consumidores para ser productores y participes de los contenidos. A esto se suma la posibilidad de compartir. La creación sin difusión no tiene sentido (García-Galera y Valdivia, 2014, p.10). Es así, que antes se difundían los mensajes a través de los medios de comunicación masivos, con una línea editorial marcada. Ahora, gracias a internet, los mensajes tienen otra vía, que puede llegar a la gran mayoría de ciudadanos sin necesidad de estar aprobados para ser difundidos.

Gracias a los espacios 2.0 que generan interacción entre distintos públicos, 
el ciberactivismo, activismo en línea, o como lo se lo quiera llamar, ha logrado movilizar a colectivos y sociedades que, tiempo atrás, se mantenían al margen de distintos asuntos sociales y políticos. Además, ha congregado a distintos actores sociales que antes se encontraban dispersos (Lasén y Alberniz, 2008, pp. 243-266) en pro o en contra de un ideal especialmente político.

El ciberactivismo, si bien es cierto, no ha roto todos los paradigmas dentro de las teorías de la comunicación de masas (Sierra-Caballero, 2018, p. 980) sí ha generado un espacio para el desarrollo de diversos movimientos sociales y políticos en torno a la red, en donde los debates ganan legitimidad según el grado de participación de los actores.

López, Roig y Sádaba (2003, p. 6) defienden el sentido democrático de la red, ya que se aleja del "triunvirato mediático" conformado por los medios de comunicación tradicionales, y genera nuevas agencias sociales y formas de participación colectiva.

\section{El contexto ecuatoriano: internet y política}

En los últimos 10 años, las redes fijas y móviles en Ecuador han penetrado con mayor fuerza entre la población. En cifras de la Agencia de Regulación y Control de las Telecomunicaciones (Arcotel, 2019) las tasas de internet fijo en 2010 eran de un 3,35\%, mientras que en 2018 ascienden a $11,28 \%$, en tanto que la tasa de internet móvil en el país pasó de 2,35\% en 2010 a $53,41 \%$ en 2018, lo que representa un claro ascenso en cuanto al acceso a las redes.

Un mundo más globalizado, con mayor acceso a las distintas conexiones, ha cambiado las plazas y las calles como lugares de protesta por las redes sociales y la internet. Es así que, antes, durante y después del mandato de Rafael Correa se ha generado una amplia participación en espacios virtuales como Facebook y Twitter, que hace de las redes las principales plataformas para expresar opiniones acerca de este gobierno.

En Ecuador, desde 1996 se ha evidenciado una crisis institucional propia de la inestabilidad política con el derrocamiento de tres presidentes, lo que produjo una desconfianza en las instituciones e incluso puso en riesgo el sistema democrático. No obstante, con la llegada de Rafael Correa a la presidencia en 2007, se produce cierto clima de estabilidad gubernamental, basada en una legitimidad (mayoría) dentro de la representación del Parlamento (Asamblea Nacional) y múltiples cambios estructurales en el aparato administrativo del Estado.

Durante los tres primeros años se marcaron varios hitos en el ámbito político, como el cambio constitucional (2008), y la creación de dos nuevas funciones del Estado (Electoral, Participación Social), que se sumaron a las ya existentes (Ejecutivo, Legislativo y Judicial). La nueva Constitución trajo consigo la necesidad de aprobar nuevas leyes, como la Ley de Comunicación (LOC), Ley de Orgánica de la Administración Pública, Ley Orgánica del Servicio Civil y Carrera Administrativa, entre otras.

Fue la Ley de Orgánica de Servicio Público (LOSEP) la que generó una revuelta policial el 30 de septiembre de 2010 y generó una fractura dentro de la coyuntura política. La revuelta se generó en la mañana de lo que después se llamaría el 30-S, a raíz de una protesta policial, en la que un grupo de policías concentra- 
dos en el Regimiento Quito manifestaban su malestar por lo que serían sus condiciones laborales con la entrada en vigor de la ley antes mencionada. El día de la protesta, el entonces presidente Rafael Correa, visitó el recinto policial para hablar con los policías y esto desencadenó los acontecimientos, en los que resultaron civiles heridos y policías muertos.

Ese mismo día, el Ejecutivo, amparado en los Art. 164, 165 y 166 declaró el Estado de Excepción. Para este caso los medios de comunicación se ciñeron al Art. 165 numeral 4 de la Constitución, que señala que el Presidente o la Presidenta de la República puede: "Disponer censura previa en la información de los medios de comunicación social con estricta relación a los motivos del estado de excepción y a la seguridad del Estado" (Constitución de la República del Ecuador, 2008). Así, los medios de comunicación se abstuvieron de abrir sus vías informativas tradicionales (radio, prensa y TV) durante la mayor parte de la jornada, exceptuando los noticieros de radio y TV que pudieron transmitir sus informativos en los horarios estelares.

Este suceso no impidió que los medios de comunicación tradicionales hicieran uso de medios alternativos para llegar a sus públicos mediante sus páginas web y las redes sociales, especialmente en Twitter y Facebook, donde se llevó a cabo la mayor parte de interacción con los usuarios. Los medios tradicionales vieron en internet un aliado, ya que la normativa no indicaba una prohibición explícita de transmisión de información a través de los medios digitales, siendo estas vías las protagonistas a la hora de transmitir información.

La viralización de la protesta en un contexto donde la penetración del internet móvil no era mayoritaria, como se evidencian en las cifras de Arcotel del año 2010, es un indicador de la búsqueda de espacios de participación política por parte de los ciudadanos, quienes sentían que su voz rebasaba los espacios formales de la política y trascendía a los de la participación política virtual, en donde los retuits y las respuestas iban marcando la audiencia de cada uno de los participantes.

\section{Metodología}

Las etnografías virtuales rompen con las formas clásicas del estudio del activismo y la acción reivindicativa (Sádaba, 2012, p. 756); llevan a los observadores a campos de acción más complejos en donde la interacción y, en muchos casos, el anonimato hacen sentir al individuo libre de opinar sobre cualquier tema y, a la vez, le otorgan cierto poder (Castells, 2003, p. 2) como el de ser visualizado por otras personas dentro de la red y que sus ideas sean leídas, lo que en los medios de comunicación no puede hacerse, ya que los espacios dentro de éstos son limitados.

Este tipo de análisis mediante etnografía virtual (Hine, 2011) permite elegir una serie de datos y materiales a partir de búsquedas avanzadas, en cuyos mensajes se analizan los impactos desde una mirada cualitativa y se desentrañan los discursos que estos mensajes contienen, las distintas posturas, de dónde provienen y los argumentos. Si bien es cierto, algunas empresas e investigaciones están intentando generar análisis de sentimiento automatizado mediante grandes bases de datos (Big Data), en la realidad estas propuestas se encuentran en proceso, ya que el lenguaje natural de los sujetos presenta múltiples variantes como las ironías, sí- 
miles, retóricas, memes, etc. (Pla y Hurtado, 2013, p. 221).

Domínguez, Beauliey, Estalella, Gómez, Schnettler y Read (2007) argumentan que internet es un entorno vasto de interacciones sociales en donde confluyen distintos significados e identidades culturales, abriendo un campo a la metodología cualitativa.

El presente trabajo muestra un análisis de sentimiento de usuarios, no automatizado, dentro de Facebook y Twitter durante la revuelta policial del 30-S en Ecuador. Se escogió este hecho puntual porque marcó un punto de quiebre dentro de la política nacional; además las redes sociales tomaron por primera vez el protagonismo frente a los medios de comunicación tradicionales, debido a que se declaró Estado de Excepción, lo que prohibía a los medios emitir contenidos propios. Es así que los propios usuarios, además de emitir información, exponían su postura sobre el régimen dejando claro su posicionamiento político.

Por ello, se escogieron los portales tomando en cuenta la mayor visibilidad y el mayor número de seguidores dentro de la web, es decir, los que agrupan las mayores audiencias según la temática.

En ese momento el protagonismo se centró en el personaje de Rafael Correa. Sin embargo, desde el ámbito virtual este actor político ha rebasad el $30 \mathrm{OS}$ y es muchas veces uno de los personajes con mayor relevancia en Twitter y Facebook por su constante actividad dentro de estas redes. Incluso, pese a su salida del país luego de finalizar su mandato, sus seguidores y detractores en la red continúan siguiendo los perfiles del exmandatario, y creando nuevos perfiles de apoyo.

Este análisis de sentimiento "no automatizado" puede aportar con nuevas es- trategias al análisis cualitativo, además de proponer ejemplos el comportamiento de los usuarios de la red durante situaciones coyunturales consideradas de alto impacto. Las ventajas del uso de los medios digitales radican en la inmediatez de los mensajes y en la amplia difusión que tienen entre sus públicos. Caldevilla (2009, p. 32) dice que las jerarquías dentro de los entornos online se van difuminando de cara a la participación activa de los usuarios. En este caso, los ciudadanos son los principales creadores y consumidores de su información, una especie de prosumidores "prosumers" (Toffler, 1990).

Estas potencialidades de la red también tienen su otra cara; centrada en la total libertad de la red, ésta trae consigo una especie de revueltas o conflictos dentro de la misma, puesto que la información, en la mayoría de los casos, circula sin ser contrastada. El $30-S$ no estuvo exento de este problema, ya que tanto los detractores como los seguidores del entonces presidente Correa se cruzaron en una suerte de reyerta online, en donde cada bando exponía, desde su punto de vista, su discurso en lo referente a la sublevación policial.

Es así que este trabajo mostrará la participación de los medios de comunicación tradicionales en el entorno online, con foco en la participación que hicieron los usuarios dentro de estos perfiles. Por último, la interacción de los mismos usuarios en torno a la figura de Rafael Correa como el personaje central durante toda la jornada.

\section{Resultados}

Facebook tuvo gran protagonismo en cuanto a la defensa del mandatario, 
mediante la creación de perfiles en los que se hablaba, sobre todo, del proyecto político. Mientras que Twitter se convirtió en un medio de comunicación en línea, en el que la ciudadanía publicaba pequeñas píldoras especialmente informativas sobre la jornada.

A continuación, se verán algunos ejemplos de páginas que siguen activas hasta la actualidad y que, en su momento, fueron protagonistas durante la revuelta, todas ellas de apoyo a Rafael Correa. No se han incluido páginas de Facebook opositoras debido a que muchas de ellas se encuentran inactivas y algunas, destinadas a la publicación de bromas, han sido cerradas, como es el caso de "Crudo Ecuador" después de una presunta persecución al líder de este portal. La primera página a analizar se denomina "Yo Apoyo a Rafael Correa”.
Los perfiles de uso en esta página se acercan más a las clases medias bajas y medias, ya que, al señalar a los usuarios, Facebook muestra una breve descripción de la persona. De esta forma se puede ver que la mayoría de los seguidores más activos no posee estudios de bachillerato ni secundarios, incluso en sus descripciones personales se muestran faltas ortográficas.

La edad promedio de los usuarios de esta página es de 30 a 60 años, es decir, la mayoría dentro de la Población Económicamente Activa. Una característica es que los comentarios vertidos, por lo general, tienen un tinte "emocional". Se defiende de una manera directa el proyecto político, especialmente con un discurso de ataque al adversario, al opositor, refiriéndose a ellos como "pelucones", "los mismos de siempre", "los sufridores", entre otros. Este discurso de clase hace alusión

\section{Imagen 1}
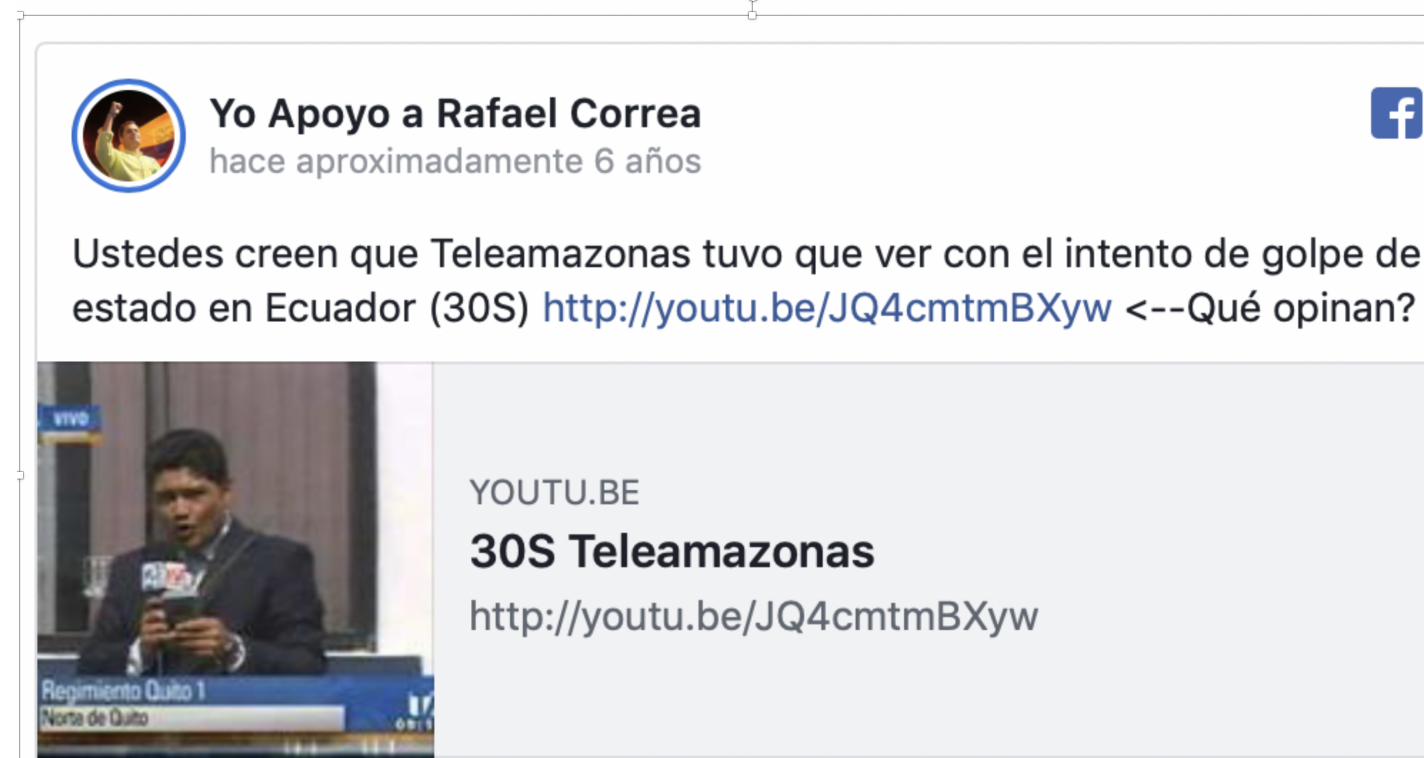

YOUTU.BE

30S Teleamazonas

http://youtu.be/JQ4cmtmBXyw

Fuente: Yo Apoyo a Rafael Correa. Recuperado de: https://www.facebook.com/YoApoyoAlMashi/posts/10151446056268494 
a aquellas élites tradicionales que, durante décadas, fueron las protagonistas en la esfera política ecuatoriana. Con el pasar de los años, las fotos de portada del sitio cambian. Sin embargo, siempre se mantienen las de Rafael Correa triunfante.

Pese a que la revuelta ocurrió en 2010, se puede observar que los usuarios siguen comentando sobre este hecho dos y tres años más tarde. El $30 S$ generó cierto rechazo a los golpes de Estado como estrategia política. En parte, este suceso produjo una consciencia democrática amparada en el sistema de elecciones libres.

En la imagen 1 se puede evidenciar un rechazo a algunos medios de comunicación, especialmente de la televisión y periódicos. Hay críticas por parte de los seguidores de esta página web en contra de lo que ellos consideran una "instigación" hacia un golpe de Estado. Durante los 10 años de gobierno de Rafael Correa la relación con los medios de comunicación no estuvo exenta de polémica. Por una parte, los medios acusaban al poder político de ejercer mordazas y censura con la Ley de Comunicación, mientras que el gobierno argumentaba que había un ataque directo al Ejecutivo y que los medios defendían los intereses de los grandes grupos económicos.

En referencia a los sucesos, el administrador de la página subía pequeñas noticias con acontecimientos al instante. De esas pastillas informativas, los seguidores comentaban, en su mayoría, en apoyo al entonces presidente y arremetían contra la Policía y la oposición. Aunque también hubo lugar para algún detractor que responsabilizaba al Gobierno de los hechos. Estos usuarios entraban en disputa con los seguidores y terminaban por dejar de comentar. Se cumplía la teoría de la Espiral del Silencio (Neuman, 1995), en el que las posiciones dominantes acallan a aquellas que son parte de las minorías. Siempre bajo la figura del antagonista, bajo el paraguas del enemigo.

Mientras que los detractores del ex presidente se concentraban en los perfiles de los medios de comunicación, los usuarios alegan una culpa directa del entonces mandatario. Los comentarios en torno a esta publicación tienen un sentimiento negativo en relación a la figura de Correa, como se observa en el titular de la imagen 2.

Los datos referidos a la participación en los perfiles de los medios de comunicación son superiores a los de otras páginas. Esto se debe a que el número de seguidores (audiencias potenciales) son mayores, debido al alcance generalista que tienen los medios en internet. Un análisis a los comentarios vertidos en el diario El Universo, uno de los más grandes del país, evidencia esa disputa del medio con el anterior gobierno.

Sin embargo, otros periodistas y blogueros independientes usaron Twitter como el medio predominante para difundir información. En ese entonces Twitter permitía mensajes con apenas 140 caracteres, por lo que todo debía ser difundido en forma de pastillas informativas inmediatas. El tono de estos mensajes es neutro. Cabe mencionar que el tuit oficial acerca de la declaratoria del Estado de Excepción tuvo más de 1.000 retuits y fue uno de los que mayor impacto tuvo durante la jornada, como lo evidencia esta búsqueda que muestra todos los mensajes generados en esa cuenta entre el 30 de septiembre y el 1 de octubre. Imagen 3

El sentimiento manejado desde esta cuenta es neutro. $\mathrm{Al}$ ser un perfil oficial no podía entrar en un clima de confrontación 


\section{Imagen 2}

\section{EC El Comercio}

"La prensa fue un instrumento de grupos políticos que buscaban la caída del Gobierno" fueron las palabras del mandatario en una entrevista. ¿Considera correcta la apreciación del mandatario al creer que la prensa estuvo tras los hechos del $30 \mathrm{~S}$ ?

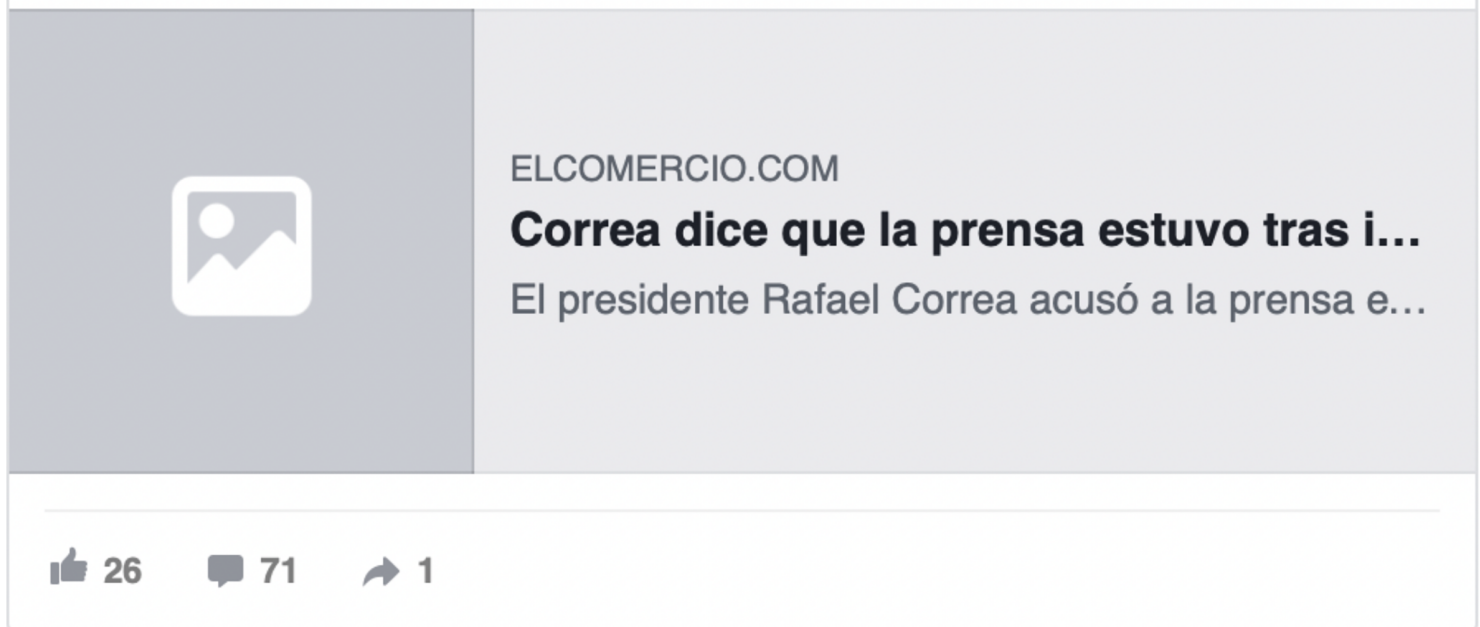

Fuente: El Comercio. Recuperado de: https://www.facebook.com/elcomerciocom/posts/178522102245333

Imagen 3

\section{Presidencia ECU}

@Presidencia_Ec

Gobierno declara estado de Excepción \#Ecuador \#30S

O 51 1:37 PM - Sep 30, 2010 
Imagen 4

\section{Radio Tarqui \\ @radiotarqui}

Nebot: Defiendo a la Democracia, un llamado al pueblo de Guayaquil y Ecuador, jamas golpistas y contemplativo con la delincuencia \#PoliciaEc

O 1 4:12 PM - Sep 30, 2010

$\therefore$ See Radio Tarqui's other Tweets

Fuente: Radio Tarqui. Recuperado de https://twitter.com/radiotarqui/status/26015181210

Imagen 5

(2) Diario Expreso

@Expresoec

\#policiaec Presidente de Perú dice que cancilleres de Sudamérica viajarán a Guayaquil para "defender la democracia"

O 2:12 PM - Sep 30, 2010

¿ See Diario Expreso's other Tweets

Fuente: Diario Expreso. Recuperado de https://twitter.com/Expresoec/status/26007133996

Imagen 6

CNN en Español

@CNNEE

El gobierno de \#Ecuador instruyó a los canales de televisión a enlazarse a la señal de la estatal Ecuador TV \#policiaec

O 2:38 PM - Sep 30, 2010

87 people are talking about this 
Textos y contextos $\mathrm{N}^{0} 20$

Imagen 7

Susana Morán

@susanamorg

RT @elcomerciocom: Cinco muertos y 193 heridos dejó jornada de ayer, según Ministro http://bit.ly/9uFnGb \#policiaec

O 3 1:27 PM - Oct 1, 2010

\section{7 people are talking about this}

Fuente: Susana Morán. Recuperado de: https://twitter.com/susanamorg/status/26104659037

con opositores. Incluso deriva la información a otro medio de comunicación gubernamental, El Ciudadano, al parecer, con el fin de no generar un conflicto entre opositores y defensores.

Si bien es cierto, los discursos se alejan de los motivos de la revuelta y se centran en la inseguridad (delincuencia) debido a que los policías no estaban custodiando las calles, las publicaciones predominantes se enfocan en el estado físico de Rafael Correa y en la estabilidad democrática. De hecho, los opositores políticos del entonces presidente (Jaime Nebot) y otros presidentes de la región (Alan García-Perú; Evo Morales-Bolivia; Hugo Chávez-Venezuela) se mantenían firmes en la defensa de la democracia y la repulsa a un posible derrocamiento.

Cabe destacar que se generó un interés particular desde el exterior en relación con este suceso. Tanto los medios internacionales como otros países de la región se hicieron eco de los hechos. Los medios de comunicación privados se engancharon a la señal de los medios oficiales y desarrollaron entrevistas con expertos en temas políticos, debido a que tampoco podían emitir contenidos propios durante la vigencia del Estado de Excepción. No obstante, sus cuentas en redes sociales se mantuvieron activas narrando los sucesos.

Con respecto a los periodistas digitales que mantuvieron informada a la audiencia, destaca @susanamorg (Ecuador), @andresgaj (Ecuador), @earcos (España), entre otros. Estos influencers únicamente hicieron un seguimiento de la jornada sin dar su punto de vista. Se nutrieron de otras fuentes y especialmente replicaron las transmisiones oficiales. Estos perfiles ganaron notoriedad a medida que la jornada avanzaba, pero además continuaron comunicando días después del suceso. Imagen 7

En la red Twitter se generó una dinámica cambiante. Había un mensaje corto nuevo de dos a cinco minutos, más rápido incluso que los flashes televisivos emitidos por CNNE, Ecuador TV o Radio Pública. Los medios transmitían la información oficial mediante esta red, mientras que los usuarios narraban lo que iba sucediendo. 
Algunos mensajes que pueden ser resaltados:

- roinujsallirav @TVesAlDia cierran bancos en Ecuador golpe de estado. Twitter - 30/09/2010 17:44:54. (SIC)

- GabiPizarro Atentos con \#Ecuador!!! Fuerzas armadas se rebelan contra el Gobierno, ¿posible golpe de Estado?, no por favor! Twitter - 30/09/2010 17:15:23. (SIC)

- jorgeramadan RT @Jrodass: RT @EcuaLink: 4 Asaltos Bancarios en \#Guayaquil !! Uno de los resultados de la Sublevación Policial en \#Ecuador \#fb. Twitter - 30/09/2010 22:51:32. (SIC) -pepezurita @Oasisantonio hay relajo más en Quito que en cualquier otro lado, en Guayaquil supuestamente muchos robos y asaltos. Twitter 30/09/2010 20:11:19. (SIC)

En la siguiente tabla se expone un resumen del sentimiento en línea que generó esta jornada. Para ello, se tomaron en cuenta dos aspectos referentes al discurso ¿qué se dice? (configuraciones narrativas) y ¿desde dónde se dice? (posiciones discursivas) (Conde, 2009). Así se puede proponer un ejemplo de cómo y en qué contextos se emiten estos mensajes.

\section{Tabla 1. Resumen sobre la valoración en línea de acuerdo con el discurso colectivos de grupos}

\begin{tabular}{|c|c|c|}
\hline Valoración & $\begin{array}{l}\text { Posición discursiva } \\
\text { (quién enuncia) }\end{array}$ & $\begin{array}{l}\text { Configuración narrativa (qué } \\
\text { se enuncia) }\end{array}$ \\
\hline Neutra & Presidencia de la República & $\begin{array}{l}\text { Sucesos relativos a la jornada. } \\
\text { Estado de salud de Rafael Correa. } \\
\text { Normativas, decretos emergentes. }\end{array}$ \\
\hline Positiva & Presidencia de la República & Defensa de la democracia. \\
\hline Neutra & $\begin{array}{l}\text { Medios de comunicación nacionales } \\
\text { e internacionales }\end{array}$ & $\begin{array}{l}\text { Difusión de contenidos emitidos } \\
\text { por los medios oficiales estatales } \\
\text { referentes a la jornada. } \\
\text { Entrevistas a expertos políticos. }\end{array}$ \\
\hline Neutra & Opositores políticos & $\begin{array}{l}\text { Restitución del orden democrático } \\
\text { y rechazo a un posible } \\
\text { golpe de Estado. }\end{array}$ \\
\hline Negativa & $\begin{array}{l}\text { Perfiles críticos a Hugo Chávez y } \\
\text { Fidel Castro }\end{array}$ & $\begin{array}{l}\text { Hablan de una posible injerencia } \\
\text { del régimen cubano y venezolano } \\
\text { en la situación nacional. }\end{array}$ \\
\hline Neutra & Periodistas digitales & $\begin{array}{l}\text { Siguen la coyuntura especialmente } \\
\text { proveniente de otros periodistas y } \\
\text { de medios oficiales. }\end{array}$ \\
\hline Positiva & $\begin{array}{l}\text { Defensores de Hugo Chávez y Fidel } \\
\text { Castro }\end{array}$ & $\begin{array}{l}\text { Muestran su apoyo a Rafael Correa } \\
\text { y hablan de un golpe de Estado } \\
\text { planificado. }\end{array}$ \\
\hline
\end{tabular}




\section{Conclusiones y reflexiones}

Este apartado pretende ser, más que un espacio de conclusiones, una tribuna de reflexiones finales, mediante el cual se pretende poner en evidencia aspectos como la importancia de la comunicación en línea en los procesos políticos y en la movilización social, sin importar las ideologías políticas o posiciones sociales.

La red, al ser uno de los pocos medios democratizados y democratizantes, como lo explica Gimmler (2001, p. 22), en el que los participantes están en igualdad de condiciones para proponer los temas y mostrar a la esfera pública sus ideas y opiniones, rompe con la Agenda Setting (McCombs y Shaw, 1972) con la que los medios de comunicación decidían los temas mediante el orden de importancia, seleccionando aquellos que seguían los intereses y la línea editorial del medio o aquellos que se creía podían vender más.

Es así que, con la irrupción masiva de internet y de las redes sociales, los papeles cambian. Pese a que los grandes holdings mediáticos no han perdido su poder, se apoyan en contenidos que circulan por la red tanto para llamar la atención de los públicos, así como para no quedarse rezagados de los temas de actualidad.

Se ha pasado de una sociedad de consumidores mediáticos a una de prosumidores (Toffler, 1990), en la que los ciudadanos son más activos con los contenidos que quieren ver, leer, escuchar y divulgar. Este aspecto se inscribe también en la vida política. Los sujetos no buscan ser únicamente votantes, sino que se interesan cada vez más por lo que pasa en sus barrios, en las administracio- nes, usan este tipo de plataformas para mostrar su agrado o descontento. Pese a que la actividad en línea es mayor entre los jóvenes, ya que ellos han vivido más este proceso (Prensky, 2001, p. 9) cabe destacar que las personas de mediana edad (35 a 55 años) empiezan a adaptarse a las nuevas tecnologías y empiezan a participar dentro de la web 2.0, muchas veces de forma autodidacta, otras mediante cursos o con ayuda de sus hijos o familiares menores.

Volviendo la vista hacia atrás, Ecuador ha vivido en la última década un período de relativa estabilidad política, luego de haber tenido tres derrocamientos en menos de diez años, en los cuales la sociedad civil se organizaba a través de llamadas telefónicas u otras vías. Los medios de comunicación eran considerados omnipresentes en algunos de los discursos más críticos. Sin embargo, esta omnipresencia y omnipotencia se pone en entre dicho cuando entran en la esfera las redes sociales. Las dinámicas y las narrativas cambian, los usuarios aprovechan esa "indignación" para demostrarla mediante estas plataformas. No necesitan acceder a los medios de comunicación porque ya lo hacen desde las propias redes.

En el caso particular del 30S, se generó un juego de antagonistas, de defensores y detractores, explotó en una actitud de visceralidad en donde lo emocional predominó frente a lo racional.

Con respecto a la técnica, si bien sería interesante aplicar este tipo de análisis al manejo de grandes bases de datos mediante la aplicación automática del Big Data, aun no es posible recuperar información de años atrás (8 años), lo cual limita que algunos de estos estudios utilicen las búsquedas avanzadas, ya que 
el mineo de los datos hay que hacerlo casi al instante. Por ello, se recomienda realizar el levantamiento de datos virtuales a los pocos días de sucedidos los hechos para recopilar la mayor información posible. 


\section{Bibliografía}

Aibar, E. (1996). La vida social de las máquinas: orígenes, desarrollo y perspectivas actuales en la sociología de la tecnología. REIS, 141-170.

Agencia de Regulación y Control de las Telecomunicaciones, Arcotel [en línea] (2018). Servicio de Acceso a Internet. Recuperado de: http://www.arcotel.gob.ec/estadisticas-2/

Bauman, Z. y Lyon, D. (2013). Vigilancia líquida. Barcelona: Paidós

Caldevilla, D. (2009). Democracia 2.0: La política se introduce en las redes sociales. Pensar la publicidad, 3(2), 31.

Campos Freire, F. (2008). "Las redes sociales trastocan los modelos de los medios de comunicación tradicionales". Revista Latina de comunicación social, 11(63).

Castells, M. (1999). La sociedad red. La era de la información: economía, sociedad y cultural. Madrid: Alianza Editorial.

Castells, M. (2000). Prefacio: "Autocomunicación de masas y movimientos sociales en la era de Internet". En Manuel Castells (UOC y University of California, Los Ángeles). Anuario del Conflicto Social, 1(1).

Castells, M. (2003). "Internet, libertad y sociedad: una perspectiva analítica". Polis. Revista Latinoamericana, (4).

Constitución de la República del Ecuador (2008). Asamblea Nacional del Ecuador. Quito: Registro Oficial, 449.

Del Álamo, F. (2009). Análisis sociológico del sistema de discursos. Madrid: CIS,

De La Cueva, J. (2018). "Internet como entorno de la opinión pública: envolviendo los derechos fundamentales en derechos ordinarios". Revista internacional de pensamiento político, 7, 93-115.

Domínguez, D., Beaulieu, A., Estalella, A., Gómez, E., Schnettler, B., y Read, R. (2007, Noviembre). Etnografía virtual. En Forum Qualitative Sozialforschung/Forum: Qualitative Social Research (Vol. 8, No. 3, pp. 3-07).

Eco, U. (1999). Apocalípticos e Integrados. Barcelona: Editorial Lumen-Tusquest.

García-Galera, M., y Valdivia, A. (2014). "Prosumidores mediáticos: Cultura participativa de las audiencias y responsabilidad de los medios". Comunicar: Revista científica iberoamericana de comunicación y educación, (43), 10-13.

Gil Ramírez, H., y Guilleumas García, R. (2017). "Redes de comunicación del movimiento 15M en Twitter". Redes: revista hispana para el análisis de redes sociales, 28(1), 0135-146.

Gimmler, A. (2001). "Deliberative democracy, the public sphere and the Internet". Philosophy \& Social Criticism, 27(4), 21-39.

Hill, K., y Hughes, J. (1998). Cyberpolitics: Citizen activism in the age of the Internet. Nueva York: Rowman \& Littlefield Publishers, Inc.

Hine, C. (2011). Etnografía virtual. Barcelona: Editorial UOC.

Lasén, A., y Martínez de Albéniz, I. (2008). "Movimientos, movidas y móviles: un análisis de las masas mediatizadas". En Sádaba, Igor y Gordo, Ángel (coords.): Cultura Digital y Movimientos Sociales. Madrid: La Catarata.

Latour, B. (2005). Reassembling the social-an introduction to actor-network-theory. Reassembling the Social-An Introduction to Actor-Network-Theory. Oxford: Oxford University Press.

López, S., Roig, G., y Sádaba, I. (2003). Nuevas tecnologías y participación política en tiempos de globalización. Bilbao, España: Hegoa.

Lozada, M. (2004). "El ciberciudadano: representaciones, redes y resistencias en Venezuela y América Latina". Políticas de ciudadanía y sociedad civil en tiempos de globalización. Caracas: FACES, Universidad Central de Venezuela, 167. 180 
Mattelart, A., y Mattelart, M. (1997). Historia de las teorías de la comunicación. Barcelona: Ediciones Paidós Ibérica. Mc'Combs, M., y Shaw, D. (1972). "The agenda-setting function of mass media". Public opinion quarterly, 176-187. Noelle-Neumann, E. (1995). La espiral del silencio. Barcelona: Paidós.

Pla, F., y Hurtado, L. (2013, Septiembre). ELiRF-UPV en TASS-2013: Análisis de sentimientos en Twitter. En XXIX Congreso de la Sociedad Española para el Procesamiento del Lenguaje Natural (SEPLN 2013). TASS (pp. 220-227).

Prensky, M. (2001). "Nativos digitales, inmigrantes digitales". On the horizon, 9 (6).

Sádaba, I. (2012). "Acción colectiva y movimientos sociales en las redes digitales. Aspectos históricos y metodológicos". Arbor188 (756), 781-794

Sassen, S. (2010). Territorio, autoridad y derechos (Vol. 3067). Buenos Aires: Katz Editores.

Sierra Caballero, F. (2018). Ciberactivismo y movimientos sociales. El espacio público oposicional en la tecnopolítica contemporánea. Revista Latina de Comunicación Social, 73, 980-990.

Tavares, W., y Pineda, C. (2018). "Participación democrática en Internet y establecimiento de las redes sociopolíticas virtuales". Praxis sociológica, (23), 41-63

Toffler, A. (1990). La tercera ola. Espuglets de Llobregat: Plaza \& Janés.

Van Dijk, T. (2003). Ideología y discurso: una introducción multidisciplinaria. Barcelona: Grupo Planeta (GBS). 